\title{
Gute Arbeit und ökologische Innovationen
}

\section{Neu erschienen: Guido Becke (Hrsg.): Gute Arbeit und ökologische Innovationen. Perspektiven nachhaltiger Arbeit in Unternehmen und Wertschöpfungsketten. München 2019, 323 S., ISBN 978-3-96238-118-9. Oekom Verlag}

\section{Eva Senghaas-Knobloch ${ }^{1}$}

Online publiziert: 17 . Juli 2020

c) Der/die Autor(en) 2020

Die inter- und transdisziplinäre Arbeitswissenschaft hat sich, wie in dieser Zeitschrift demonstriert, schon oft besonders brisanter Themen angenommen, lange bevor sie - wie gegenwärtig die Arbeitsbedingungen in der Fleischindustrie und in den Pflegeberufen - in einer breiten Öffentlichkeit die nötige Aufmerksamkeit fanden. Ein dementsprechend pionierhafter Band wurde von dem Bremer Arbeitsforscher Guido Becke herausgegeben. Er beleuchtet systematisch und anhand konkreter Forschungsergebnisse Ansätze, in denen zugleich gute Arbeit und ökologische Innovationen angestrebt werden sowie Faktoren, die eine Umsetzung begünstigen bzw. verhindern. Dies ist eine Forschungsthematik, die bisher kaum behandelt wurde, jedoch in Zeiten des Klimawandels und der VN-Nachhaltigkeitsziele dringend der Beachtung bedarf. In dem Band wird aufgezeigt, dass sich Fragestellungen aus ökologischer Sicht und aus der Perspektive der Arbeitsforschung wechselseitig befruchten können, um zu einem integrativen, normativ begründeten Gestaltungskonzept zu gelangen.

Der Band entstand auf Basis grundlegender konzeptioneller und empirischer Vorarbeiten des Herausgebers und weiterer umfangreicher Forschungsvorhaben. In zwei grundlegenden Kapiteln verdeutlicht Becke eingangs das Wechselverhältnis von Arbeit und Ökologie unter besonderer Berücksichtigung des ressourcen- und fähigkeitsorientierten Verständnisses von menschlicher Arbeit, wie es im Bericht über Arbeit und menschliche Entwicklung der VN-Organisation für Entwicklung (UNDP) 2015 als Rahmenkonzept entfaltet wurde. Der UNDP-Bericht geht

Prof. i.R., Dr. Eva Senghaas-Knobloch

esk@uni-bremen.de

1 Forschungszentrum Nachhaltigkeit, Universität Bremen, Bremen, Deutschland
- so wie auch in der Arbeitswissenschaft diskutiert - von einem erweiterten Arbeitsbegriff aus und versteht nachhaltige Arbeit als eine Tätigkeit, die menschliche Entwicklung fördert, aber unerwünschte Nebenwirkungen und nachteilige Folgen dieser Tätigkeiten verringert oder beseitigt. Dies Rahmenkonzept wird von Becke genutzt, um vier Gestaltungsebenen anzusprechen, zu denen die Kapitel des Buchs beitragen: die Ebenen der Gesellschaft, der Unternehmen, der Unternehmenskooperation in Lieferketten und der regionalen Kooperation.

Als Brücke zwischen dem integrativen Rahmenkonzept der UNDP und nachhaltiger Arbeit im Unternehmenskontext stellt Becke sein Konzept der ,arbeitsökologischen Innovationen" vor. Dabei geht es um neue soziale Praktiken, Methoden, Technologien und Verfahren, mit deren Hilfe gute Arbeitsqualität und ökologische Verbesserungen miteinander verknüpft werden. Arbeitsökologische Innovationen werden unter drei Gesichtspunkten betrachtet: Ressourcenregeneration, Zukunftsoffenheit und Reflexivität. Aus der Sicht der Arbeitsforschung ist es besonders relevant, dass die Ressourcenregeneration sowohl die Schonung, Erhaltung und Erneuerbarkeit natürlicher außermenschlicher Ressourcen als auch die spezifischen Ressourcen betrifft, die in psycho-physischer, persönlicher und sozialer Hinsicht das Arbeitsvermögen der Menschen als Natur- und Sozialwesen prägen. Dazu gehören auch sozial-ökologische Sinnansprüche.

Auf der Ebene der Gesellschaft argumentiert Warsewa, dass gegenwärtig der Strukturwandel der Arbeit und die geltenden arbeitspolitischen Leitbilder das wichtige sozialintegrative Potential der Arbeit für gesellschaftlichen Zusammenhalt eher schwächen. Jochum und Matuschek befassen sich mit Überlagerungen großer industriegesellschaftlicher Trends - von der Demografie bis zur Digitalisierung; sie analysieren die materiell-energetischen Grund- 
lagen der Digitalisierung, die damit zusammenhängenden zentralen Konflikte und Risiken und erörtern Möglichkeiten für eine ,digital gestützte Humanisierung der Arbeitswelt“. Von Jorck beschreibt im Zusammenhang mit der Flexibilisierung von Arbeit neue paradoxe Effekte einer Zeitnot durch „Zeit-Rebound-Effekte“ aufgrund von Beschleunigungswirkungen und diskutiert mögliche Gestaltungsansätze zu ihrer Überwindung.

Dass es bei großen politischen Veränderungsstrategien z.B. zur Emissionsreduktion oder der umfassenden Digitalisierung einer bewussten Förderung arbeitsökologischer Sichtweisen bedarf, um dem dreidimensionalen Konzept ökonomischer, ökologischer und sozialer Nachhaltigkeit gerecht zu werden, zeigen Brandl, Littig und Zielinska im Kontext von Wien auf, Friemer in der Bremer Region. Dabei wird u. a. deutlich, dass ohne Beachtung der verschiedenen Lebenswirklichkeiten von Frauen und Männern soziale Risiken und Probleme der Arbeitsqualität verschärft werden.

Auf der Ebene der Unternehmen setzen sich Süßbauer und Kolleginnen mit Ergebnissen ihrer Forschung auseinander, in der es darum geht, wie alltägliches, außerberufliches Erfahrungswissen von Beschäftigten für ökologische Verbesserungen im Betrieb wahrgenommen und eingebracht werden kann. Harrach, Schrader und Buhl stellen ihre Forschungserfahrungen mit der Innovationsmethode „Design Thinking“ und dem psychologischen „Empowerment-Konzept" im Kontext unternehmensbezogener Nachhaltigkeitsinnovationen dar. Blazejewski und Barth zeigen die diversen subjektiven Verarbeitungsstrategien, mit denen ökologisch hochengagierte Mitarbeiter auf die sehr verschiedene Resonanz ihres Engagements in Unternehmenskontexten reagieren. Die Beiträge von Roller und Fingerhut befassen sich mit Forschungsergebnissen zur arbeitsbezogenen Mobilität; dabei erweist sich, dass ökologische Lösungen für Mobilität auch beiläufig zustande kommen können, wenn Unternehmen daran interessiert sind, fachlich versierte Mitarbeiter zu halten, z. B. durch sach-materielle Unterstützung von Wochenendpendlern. Während ökologische Verbesserung durch solche Unterstützung gewissermaßen ein Beiprodukt war, wird am Beispiel alltäglicher Arbeitsmobilität aufgezeigt, dass eine ökologisch bewusste Steuerung notwendig und möglich ist, wenn entsprechende strukturelle Voraussetzungen im Betrieb dafür geschaffen sowie Arbeitszufriedenheit und eine generationssensible Innovationskultur gefördert werden.
Auf der Ebene der Unternehmenskooperationen wird von Knüpffer und Albrecht zur Optimierung von sozialen und ökologischen Aspekten in Wertschöpfungsketten mit dem „Social Life Cycle Assessment“ eine Methode dargestellt, die sich an die etablierte Methodik der Ökobilanz anlehnt und den Vorteil hat, dass sie sich an internationalen normativen Regelwerken für globale Wertschöpfungsketten orientiert. Forschungsergebnisse einer Kombination der Ökobilanzmethodik mit einem arbeitsökologischen Bewertungskonzept stellen auch Steinfeldt und Nickel vor; sie beziehen sich dabei auf das besonders für KMU so wichtige Feld der Produktinnovationen. In welcher Weise die hier geforderte unternehmensinterne Flexibilität mithilfe regelmäßiger unternehmensübergreifender ,,arbeitsökologischer Projektreviews" auch zu einer resilienten Unternehmenskooperation beitragen kann, thematisieren Becke und Nickel in einer Metaanalyse des großen Bremer Pilotprojekts.

Die Autorinnen und Autoren des Bandes setzen sich forschungsbasiert und auf hohem Niveau mit der höchst komplexen Aufgabenstellung eines integrativen Gestaltungskonzepts nachhaltiger Arbeit auseinander. Es ist zu wünschen, dass der Band breit rezipiert wird und den Anstoß zu einer neuen Forschungstradition gibt, in der trotz notwendiger Differenziertheit auch die notwendige Syntheseleistung nie aus den Augen verloren wird. Das Thema nachhaltiger Arbeitsqualität als integraler Bestandteil gesellschaftlicher Nachhaltigkeitsstrategien bedarf besonderer Förderung in Wissenschaft und Praxis.

Funding Open Access funding provided by Projekt DEAL.

Open Access Dieser Artikel wird unter der Creative Commons Namensnennung 4.0 International Lizenz veröffentlicht, welche die Nutzung, Vervielfältigung, Bearbeitung, Verbreitung und Wiedergabe in jeglichem Medium und Format erlaubt, sofern Sie den/die ursprünglichen Autor(en) und die Quelle ordnungsgemäß nennen, einen Link zur Creative Commons Lizenz beifügen und angeben, ob Änderungen vorgenommen wurden.

Die in diesem Artikel enthaltenen Bilder und sonstiges Drittmaterial unterliegen ebenfalls der genannten Creative Commons Lizenz, sofern sich aus der Abbildungslegende nichts anderes ergibt. Sofern das betreffende Material nicht unter der genannten Creative Commons Lizenz steht und die betreffende Handlung nicht nach gesetzlichen Vorschriften erlaubt ist, ist für die oben aufgeführten Weiterverwendungen des Materials die Einwilligung des jeweiligen Rechteinhabers einzuholen.

Weitere Details zur Lizenz entnehmen Sie bitte der Lizenzinformation auf http://creativecommons.org/licenses/by/4.0/deed.de. 